\title{
On-site Method for Beef Detection Based on Strand Exchange Amplification
}

\author{
Xuejiao WANG, * Chunyu YAN,* Manman WeI,* Chao SHI,** Shuyan NiU,* and Cuiping MA*† \\ *Shandong Provincial Key Laboratory of Biochemical Engineering, College of Chemistry and Molecular \\ Engineering, College of Marine Science and Biological Engineering, Qingdao University of Science and \\ Technology, Qingdao 266042, PR China \\ **College of Life Sciences, Qingdao University, Qingdao 266071, PR China
}

\begin{abstract}
Meat screening plays a significant role in human health and religion. But the identification methods for beef were little reported. In this work, a simple colorimetric method based on denaturation bubble-mediated strand exchange amplification (SEA) was developed for the rapid and sensitive identification of beef. The whole strategy was performed on a portable metal bath and the distinguishable color between positive and negative controls was observed directly by the naked eyes. The feasibility using crude extraction samples by a heating treatment in PBS for 2 min was evaluated in duck spiked by beef. The result demonstrated that the developed method could identify as low as $1 \%(\mathrm{w} / \mathrm{w})$ beef/duck within 50 min. Meanwhile, the results showed the method had a good repeatability and specificity. Therefore, this assay allows for the rapid, sensitive, specific detection of beef, and can be recommended as an effective, promising strategy for on-site meat identification.
\end{abstract}

Keywords Strand exchange amplification, colorimetric method, meat detection, beef detection, on-site detection

(Received September 25, 2018; Accepted November 8, 2018; Advance Publication Released Online by J-STAGE November 16, 2018)

\section{Introduction}

Meat, as a source of multiple proteins, is one of the most important components in human daily diet. The safety of meat not only relates to the development of the food industry, but also plays a great significance role in public health and religious factors. Recent scandals about beef safety have been reported. In a previous study, sausage samples that were labeled as containing $5 \%$ beef meat but finally no beef was identified. ${ }^{1}$ However, it cannot be determined whether is the insufficient detection limit of the method. Again, adulteration with beef was illegitimate because cattle is a national belief in large part of India $^{2}$ and the Miao nationality of China. Thus, meat screening before meat entering the market is particularly important. Current meat screening methods are not suitable for simple, rapid and on-site identify use. ${ }^{3}$ Therefore, to offer best protection, it is necessary to develop a rapid and sensitive method for on-site beef identification.

Presently, Cristina et al. ${ }^{3}$ investigated whether near infrared (NIR) can be considered as fast and highly reliable support for species identification. However, it needs the fused UV-vis-NIRMIR data matrix to improve the results, leading to timeconsuming and instrument-dependent. Also a high-performance liquid chromatography (HPLC) based method ${ }^{5,6}$ had shown precise requirements for DNA samples, and required considerable operator skills. Thus the on-site use of those methods $^{7-9}$ for rapid beef detection was limited.

$\dagger$ To whom correspondence should be addressed.

E-mail: mcp169@163.com
Compared with NIR and HPLC-based methods, nucleic acid amplification has obvious advantage, such as simple, rapid, highly sensitivity and great specificity. ${ }^{10}$ Various methods based on nucleic acid amplification were used for beef identification, such as multiplex real-time $\mathrm{PCR},{ }^{11}$ the real-time polymerase chain reaction (RT-PCR), ${ }^{12}$ digital dPCR, ${ }^{13}$ and common primer multiplex-PCR (CP-M-PCR). ${ }^{14}$ However, these methods based on PCR amplification still require expensive equipment and consume time. More recently, loop-mediated isothermal amplification (LAMP) ${ }^{15-20}$ was developed, which can react at a constant temperature and does not need any thermal cycler. Rajib et al. ${ }^{2}$ developed a LAMP assay for visually identifying cow components in meat samples. However, the whole detection, including DNA extraction, needed $1 \mathrm{~h}$ and $40 \mathrm{~min}$. Minhaz et al. ${ }^{21}$ have described a meat species identification method based on LAMP amplification and electrochemical detection. It took less than 65 min for genomic DNA detection, but it required complicated electrochemical devices. Furthermore, LAMP required four primers to ensure its specificity, resulting in the difficulty of primer design. Besides, the LAMP reaction assay can easily be affected by carryover contamination due to its high sensitivity. ${ }^{22}$

Herein, we carried out beef identification using strand exchange amplification (SEA), a novel nucleic acid detection technology established in $2016,{ }^{23}$ which relies on the dynamic dissociation of dsDNA at the reaction temperature to created single-stranded denaturation bubbles. Shown as the amplification phase in Fig. 1, under thermophilic conditions, once the complementary area of primers turned into a single strand state, the primer will specifically recognize the area by base pairing, and gain the extension at the presence of DNA polymerase. 


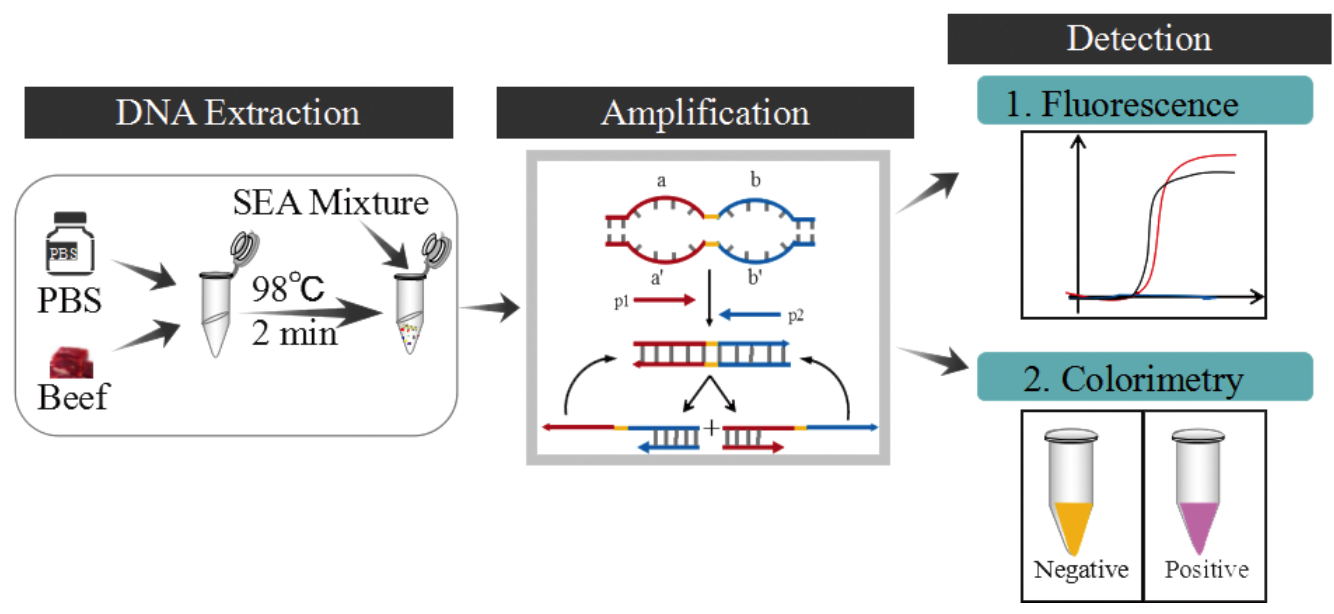

Fig. 1 Schematic illustration of beef detection based on the SEA method.

The method only needs a pair of primers. Also considering that a denaturation step by heating is not required for SEA, the reaction can be carried out at a constant temperature. Thus the SEA reaction can be performed without the need of a thermal cycler, which greatly reduces the assay cost and makes it time efficient. In addition, the nucleic acid amplification process occurred along with the produce of products, ${ }^{24}$ which can change the original reaction system and make the indicator that is presented in the reaction mixture at the beginning a color change. Meanwhile, shown as the DNA extraction phase in Fig. 1, visual detection can be realized using a crude DNA lysis mixture from a 2-min heating treatment in phosphate buffer saline (PBS). In brief, we combined the simple lysate technology with a colorimetric SEA strategy to complete the rapid and on-site identification of raw beef, which only needs a simple heating block.

\section{Experimental}

\section{Reagents and chemicals}

The primers (Table 1) used in this study were synthesized and purified by Sangon Biotech (Shanghai, China). The colorimetric and fluorescent detection kit were provided by Qingdao Navid Biotechnology Co., Ltd. (Qingdao, China). 6× DNA loading buffer, 20 bp DNA marker, ethidium bromide were purchased from Sangon Biotech (Shanghai, China). The raw meat used in this study was purchased from local supermarket.

\section{Apparatus}

A metal bath was purchased from Coyote Biotech (Pad3$100 \mathrm{C}$, China). All pictures were taken by an iphone $6 \mathrm{~s}$.

\section{Sample preparation}

Genomic DNA of beef was extracted by using the TIANamp feedstuff Animal DNA kit (Beijing, China). In addition, after homogenizing for $1 \mathrm{~mm}^{3}$ raw beef, meat mixtures were heated with $20 \mu \mathrm{L}$ PBS at $98^{\circ} \mathrm{C}$ for $2 \mathrm{~min}$, and the supernatant was directly used as the target for isothermal amplification.

\section{Amplification and detection}

Primers extended from $5^{\prime}$ to $3^{\prime}$ under the polymerization function of Bst 2.0 WarmStart DNA polymerase. The reaction temperature was optimized at $59,60,61$ and $62^{\circ} \mathrm{C}$. During the
Table 1 Sequence of oligonucleotides used in this study

\begin{tabular}{llcc}
\hline \multirow{2}{*}{ Name } & $\begin{array}{c}\text { Sequence } \\
\left(5^{\prime} \rightarrow 3^{\prime}\right)\end{array}$ & $\begin{array}{c}\text { GenBack } \\
\text { accession No. }\end{array}$ & $\begin{array}{c}\text { Length/ } \\
\text { bp }\end{array}$ \\
\hline Target & CAAAGATACCCTCTCGACTAAA- & AY676859.1 a & 47 \\
\cline { 2 - 3 } & \multicolumn{2}{c}{ CAACCAAGATAGAATAAAACA- } & \\
P1 & CAAAC & 18 \\
P2 & GTTTTGTACCCTCTCGAC & 25 \\
\hline
\end{tabular}

a. GenBank accession number.

amplification reaction, fluorescent signals were detected by the CFX Connect ${ }^{\mathrm{TM}}$ Real Time PCR System (Bio-Rad, CA, USA). The colorimetric result was visual and observed by the naked eyes.

\section{Results and Discussion}

\section{The design of SEA for beef detection}

The SEA reaction was performed by the extension of a pair of primers in the presence of DNA polymerase. To ensure the more precise and specific detection of beef, an appropriate endogenous reference gene is necessary. In this work, primers for the SEA reaction were designed from the hyperconservative region of Bos taurus Y676859.1 (Table 1). Though a specificity test was made using eleven other species, no cross reaction was observed, and thus primers designed here displayed good specificity (Fig. S1). The reaction temperature was optimized based on the $\mathrm{Tm}$ value of the primers, and $59^{\circ} \mathrm{C}$ was chosen as the most optimal reaction temperature (Fig. S2).

\section{The feasibility of beef detection by SEA method}

Genomic DNA and a DNA lysis mixture by a heating treatment were used as templates to test the feasibility of the SEA method. As shown in Fig. 2A, a significant difference was found in the fluorescence signal between the genome as a target and the negative control. The corresponding products were analyzed by $12.5 \%$ native polyacrylamide gel electrophoresis (PAGE). Also, there was a 47-bp amplification band in line 1, which was consistent with the expected length, indicating that beef was successfully detected by the SEA method. More 
A

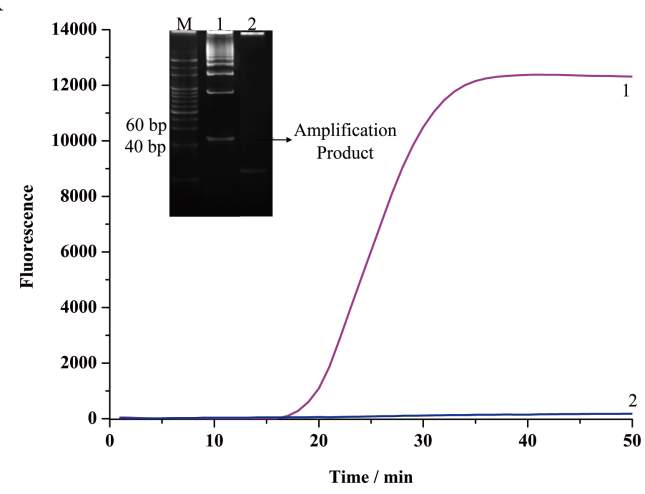

B

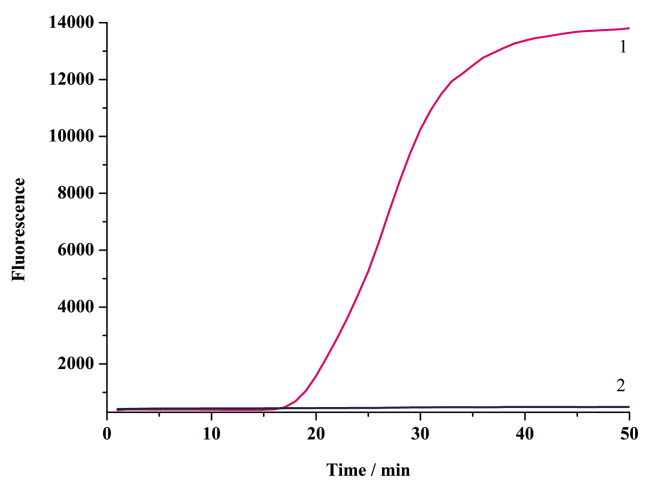

Fig. 2 (A) Feasibility of SEA to detect beef using genomic DNA as a template. $1-2$ respectively represents genomic DNA and no template control (NTC). The insert represents $12.5 \%$ native PAGE of the corresponding amplification reaction products. (B) Feasibility of SEA method to detect beef from the crude DNA by a 2-min heating treatment. $1-2$ respectively represents crude DNA by a 2-min heating treatment and NTC. Herein, mixed with $20 \mu \mathrm{L} \mathrm{PBS}, 1 \mathrm{~mm}^{3}$ beef was heated at $98^{\circ} \mathrm{C}$ for $2 \mathrm{~min}$.
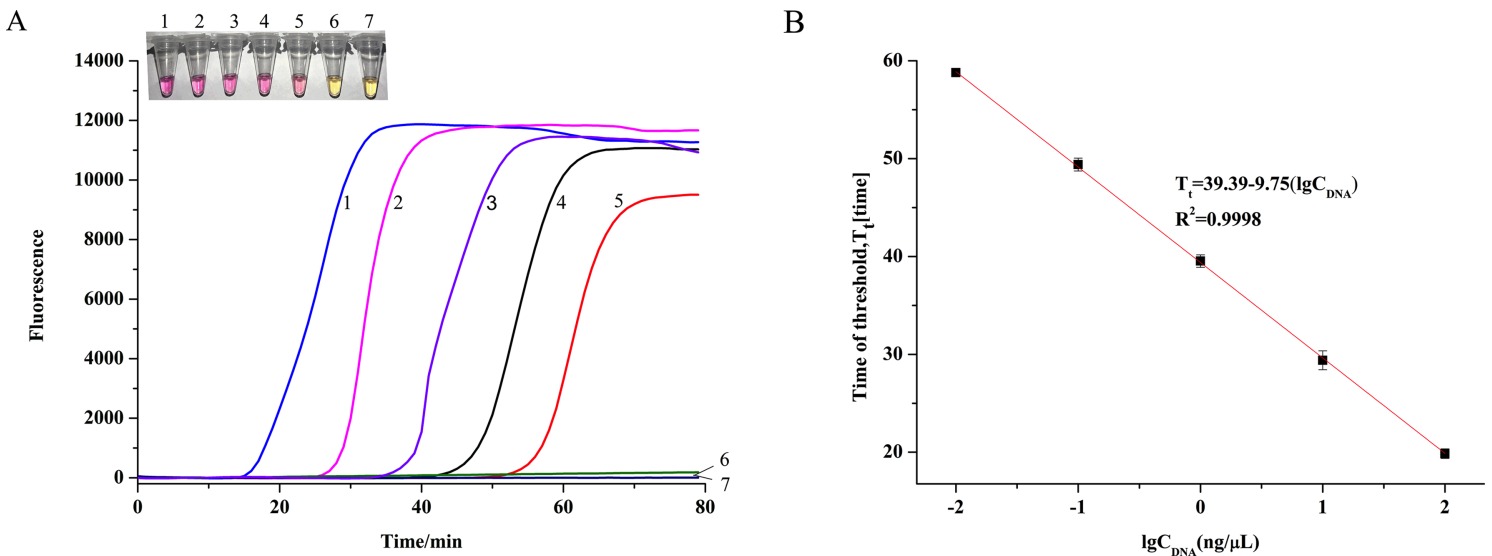

Fig. 3 (A) Sensitivity of the SEA method to detect beef genomic DNA. $1-7$ respectively represents from $100 \mathrm{ng} / \mu \mathrm{L}$ to $1 \mathrm{pg} / \mu \mathrm{L}$ with 10 -fold diluted series; 7 represents NTC. (B) Relationship between the $T_{\mathrm{t}}$ values and the logarithmic values of different concentration of targets. Error bars showed the mean standard deviations of three determinations.

importantly, the crude DNA obtained from 2 min of a heating treatment in PBS could also be used as template for SEA reaction, as observed the amplified band in PAGE gel (Fig. 2B). Besides, the repeatability of SEA was also evaluated (Fig. S3), and a good stability was observed for the beef identification. From the above results, we could come to the conclusion that SEA could realize real-time fluorescence detection of beef.

\section{The sensitivity of the SEA method to detect beef}

The sensitivity of the SEA method was evaluated by testing different concentrations of beef genomic DNA with a 10-fold diluted series. As shown in Fig. 3A, SEA could detect as low as $10 \mathrm{pg} / \mu \mathrm{L}$ of genomic DNA from beef based on real-time fluorescent signal, which was consistent with the result from colorimetric detection (Fig. 3A insert). A linear relationship was observed at a concentration range of $100 \mathrm{ng} / \mu \mathrm{L}-10 \mathrm{pg} / \mu \mathrm{L}$, shown in Fig. 3B, with the linear equation $T_{\mathrm{t}}=39.39-$ $9.75\left(\lg C_{\mathrm{DNA}}\right)$. The results showed a promising sensitivity of this method.

Currently, the developed methods for visual meat identification were based on either ELISA or UV light. Since UV light is harmful to the human body, it would cause itchy, dandruff, even cancer and skin tumor even under short-time exposure. ${ }^{15}$ For an ELISA-based method, an expensive enzyme is required, and thus increasing the detection cost. ${ }^{25}$ Herein, the result was directly observed by the naked eyes, ensuring the safety of the detection assay. In addition, the color difference between the positive (bright red) and negative (yellow) results were much easier for result judgement than the visual detection results reported by Ran et al. ${ }^{20}$

\section{Specificity of SEA to detect beef}

The specificity of SEA detection was investigated by detecting beef and four other meat species. As can be observed in Fig. 4, the fluorescence signal increased significantly for Bos taurus, whereas no fluorescence signal increase was observed for four other species. The colorimetric result was also consistent with the fluorescent result (Fig. 4 insert), which further confirmed the specificity of the SEA method for beef identification. Meanwhile, it is worth mentioning that the SEA method only needs a pair of primers, which is very simple compared with at least four primers required by the LAMP reaction. The design 


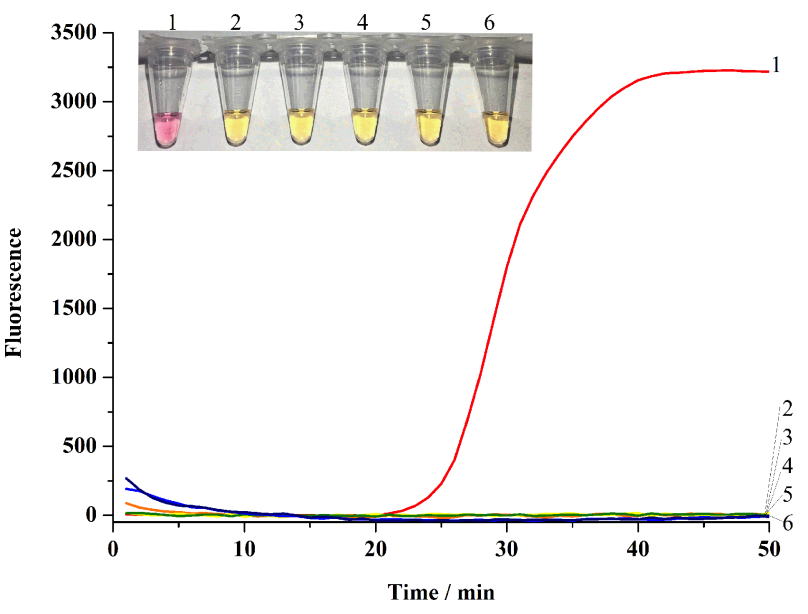

Fig. 4 Specificity of the SEA method. $1-5$ respectively represents the genome targets from Bos taurus, Sus scrofa, Ovis aries, Anatinae, S. castellani and Gallus gallus.

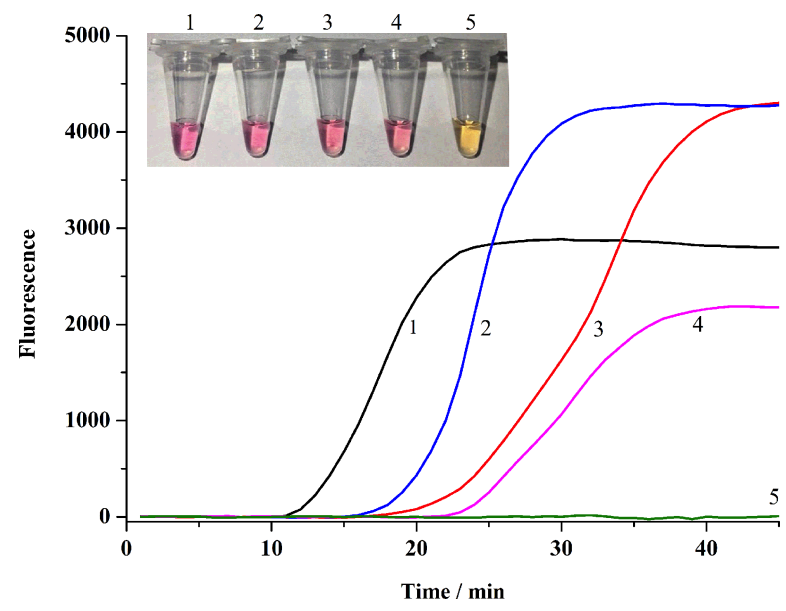

Fig. 5 On-site application of beef detection. 1-4 respectively represents the beef proportion in duck of $100,10,5 \%, 1 ; 5$ represents the NTC.

difficulty of LAMP primers limited the application on the field of highly mutant species identification.

\section{On-site application of beef detection}

Meat adulteration has been as a widely existing phenomena, which has drawn worldwide attention. Developing a simple and rapid identification method has attracted the interest of researchers in far more detail. ${ }^{25,26}$ In the global meat market, duck is the first alternative for beef adulteration due to its low price. Herein, mixtures with $100,10,5,1 \%$ (w/w) beef/duck content were prepared. In an attempt to simplify the operating steps and to shorten the assay time and to ensure the real applicability, the assay was carried out directly by testing crude DNA by a heating treatment in PBS. As indicated in Fig. 5, different proportions of beef/duck mixtures showed color changes from yellow to red, whereas the NTC maintained yellow. This result was confirmed by a significant fluorescence signal increase. Thus, the colorimetric SEA method developed in this study could identify as low as $1 \%$ beef in meat.

Taken together, the current results show that the method here is both simple and fast. A thermal cycler can be eliminated to perform the SEA reaction. The whole detection workflow from sample pretreatment to visual analysis could be completed within $50 \mathrm{~min}$, which is more time-efficient compared with about $3.4 \mathrm{~h}$ for real-time PCR ${ }^{12}$ and $100 \mathrm{~min}$ for LAMP. Most importantly, the established method could detect as low as $1 \%$ beef, which was lower than many other analysis methods, such as LESA-MS reported by Montowska et al. with a detection limit of 5\%, ${ }^{25}$ NIR reported by Meza-Márquez et al. ${ }^{27}$ and Yoshiowith et al. ${ }^{28}$ with a detection limit of $2 \%$, and equal to that of PCR methods reported by Calvo et al. ${ }^{29}$ Although the detection limit of meat contamination identification was not as low as LAMP, ${ }^{30,31}$ the method was sufficient for on-site meat contamination identification since the contamination proportion below $3.0 \%$ was considered to be unintentional contamination, and can be label-free. ${ }^{32,33}$

\section{Conclusions}

This work reported a colorimetric SEA assay for the on-site identification of beef. The whole detection workflow was available with only a metal bath, eliminating the use of an expensive thermal cycler, and could thus be used in resourcelimited settings. Compared with the conventional PCR assay, it was much simpler without any need of precise temperature circulation control. The results confirmed the feasibility of the method to detect beef genomic DNA extracted with kit and target simply heating treatment in PBS. The method is able to distinguish between raw beef, mutton, pork, duck, and chicken. When this approach was applied to the mixed beef and duck, as low as $1 \%(\mathrm{w} / \mathrm{w})$ beef was successfully identified. The whole assay was completed within $50 \mathrm{~min}$, indicating time-efficiency. These results indicated that the method is very suitable for the rapid and on-site identification of beef in a restricted environment.

\section{Acknowledgements}

The work was supported by the Shandong Province Natural Science Fund Major Basic Research Project (ZR2017ZC0123), and National Natural Science Foundation of China (31670868, 21675094).

\section{Supporting Information}

This material is available free of charge on the Web at http:// www.jsac.or.jp/analsci/.

\section{References}

1. A. F. Oliveira, J. Landero, K. Kubachka, A. R. A. Nogueira, M. A. Zanetti, and J. Caruso, J. Anal. At. Spectrom., 2016, 31,1034

2. R. Deb, G. S. Sengar, U. Singh, S. Kumar, R. R. Alyethodi, R. Alex, T. V. Raja, A. K. Das, and B. Prakash, Mol. Biotechnol., 2016, 58, 850.

3. V. Ruiz-Valdepenas Montiel, M. L. Gutierrez, R. M. Torrente-Rodriguez, E. Povedano, E. Vargas, A. J. Reviejo, R. Linacero, F. J. Gallego, S. Campuzano, and J. M. Pingarron, Anal. Chem., 2017, 89, 9474.

4. C. Alamprese, M. Casale, N. Sinelli, S. Lanteri, and E. Casiraghi, LWT_Food Sci. Technol., 2013, 53, 225. 
5. C. von Bargen, J. Brockmeyer, and H. U. Humpf, J. Agric. Food Chem., 2014, 62, 9428.

6. H. Zhu, S. Yang, Y. Zhang, G. Fang, and S. Wang, Anal. Methods, 2016, 8, 3747.

7. A. A. Argyri, E. Z. Panagou, P. A. Tarantilis, M. Polysiou, and G. J. E. Nychas, Sens. Actuators, B, 2010, 145, 146.

8. M. Zhao, G. Downey, and C. P. O'Donnell, Meat Sci., 2014, 96, 1003.

9. G. ElMasry, D.-W. Sun, and P. Allen, J. Food Eng., 2012, 110, 127.

10. M. Alikord, H. Momtaz, J. Keramat, M. Kadivar, and A. H. Rad, J. Food Meas. Charact., 2017, 12, 145.

11. R. Köppel, J. Ruf, and J. Rentsch, Eur. Food Res. Technol., 2010, 232, 151.

12. A. T. Perestam, K. K. Fujisaki, O. Nava, and R. S. Hellberg, Food Control, 2017, 71, 346.

13. C. Floren, I. Wiedemann, B. Brenig, E. Schutz, and J. Beck, Food Chem., 2015, 173, 1054.

14. W. Bai, W. Xu, K. Huang, Y. Yuan, S. Cao, and Y. Luo, Food Control, 2009, 20, 366.

15. N. Tomita, Y. Mori, H. Kanda, and T. Notomi, Nat. Protoc., 2008, 3, 877.

16. C. Zahradnik, R. Martzy, R. L. Mach, R. Krska, A. H. Farnleitner, and K. Brunner, Food Anal. Method., 2014, 8, 1576.

17. C. S. Ball, Y. K. Light, C. Y. Koh, S. S. Wheeler, L. L. Coffey, and R. J. Meagher, Anal. Chem., 2016, 88, 3562.

18. A. R. Cho, H. J. Dong, and S. Cho, Korean J. Food Sci. An., 2014, 34, 799 .
19. Y.-J. Li and J.-Y. Fan, BioChip J., 2016, 11, 8.

20. G. Ran, L. Ren, X. Han, X. Liu, Z. Li, D. Pang, H. Ouyang, and X. Tang, Food Anal. Method., 2015, 9, 565.

21. M. U. Ahmed, Q. Hasan, M. Mosharraf Hossain, M. Saito, and E. Tamiya, Food Control, 2010, 21, 599.

22. C. Ma, F. Wang, X. Wang, L. Han, H. Jing, H. Zhang, and C. Shi, Chem. Commun., 2017, 53, 10696.

23. C. Shi, F. Shang, M. Zhou, P. Zhang, Y. Wang, and C. Ma, Chem. Commun., 2016, 52, 11551.

24. N. Tomita, Y. Mori, H. Kanda, and T. Notomi, Nature Protoc., 2008, 3, 877.

25. M. Montowska, M. R. Alexander, G. A. Tucker, and D. A. Barrett, Anal. Chem., 2014, 86, 10257.

26. M. Kaltenbrunner, R. Hochegger, and M. Cichna-Markl, Food Chem., 2018, 269, 486.

27. O. G. Meza-Márquez, T. Gallardo-Velázquez, and G. OsorioRevilla, Meat Sci., 2010, 86, 511.

28. M. Kamruzzaman, Y. Makino, S. Oshita, and S. Liu, Food Bioprocess Technol., 2015, 8, 1054.

29. J. H. Calvo, R. Osta, and P. Zaragoza, J. Agric. Food Chem., 2002, 50, 5265.

30. Y. Shi, Y. Feng, C. Xu, Z. Xu, D. Cheng, and Y. Lu, Food Anal. Method, 2017, 10, 2325.

31. B. Ma, M. Dai, J. Fang, Y. Wu, and M. Zhang, Am. J. Food Technol., 2016, 11, 193.

32. S. Y. Lee, M. J. Kim, Y. Hong, and H. Y. Kim, Food Control, 2016, 66, 53.

33. Korea Biosafety Cleaing House, https://www.biosafety.or. kr. 